\title{
On two long lasting delusions in the history of equations
}

\author{
LAJOS KLUKOVITS
}

Abstract. Almost everybody was thought, that the 9th century Moshlem mathematician al-Khwarismi was the inventor of two powerful methods - called by him as al-jabr and al-muqabala - in solving quadratic equations. The second belief is that between Leonardo's Liber abaci and Luca Pacioli's Summa... happened nothing interesting in algebra. We will show that both beliefs are false by giving examples from the antiquity and analyzing Mediaeval Italian manuscripits.

Key words and phrases: ancient history, mediaeval history, equation.

ZDM Subject Classification: A35, H35.

\section{The two methods}

The belief that the 9th century moshlem mathematician al-Khwarismi was the inventor of the two powerful methods - called by him as al-jabr and almuqabala - in solving quadratic equations, could be based on Islamic sources, e.g. on the biography written by Haji Khalfa: "al-Khwarismi was the first Islamic author to write on the solution of problems by al-jabr and al-muqabala".

The meaning of this two expressions: al-jabr

- adding equal terms to both sides of an equation in order to eliminate negative terms,

The research was partially supported by TÁMOP-4.2.1/B-09/1/KONV-2010-0005.

Copyright (c) 2011 by University of Debrecen 


$$
\text { "tmcs-klukovits" — 2011/5/16 — 12:52 — page } 148 \text { — \#2 }
$$

- multiplying both sides of an equation by one and the same number in order to eliminate fractions;

- literal meaning: bonesetter (Remember the famous roman of Cervantes). al-muqabala

- reduction of positive terms by substracting equal amounts from both sides of an equation,

- literal meaning: comparing or posing opposite

In general, al-jabr wal muqabala means simply performing algebraic operations.

The first (light) objection based on Diophantus Arithmetica. In Book 1 the Alexandrian Greek mathematician dealt with such problems: if we know the sum (or difference) and the product of two unknown quantities, how to find them. His method - the so-called sum and difference method — was quite similar to that of the Mesopotamian scibes used in the period of the Old Babylonian Empire.

To make this objection complete analyze three problems from Mesopotamian clay tablets.

Problem 1. "length, width" (AO 8862 from Senkereh) I have multiplied length and width, thus obtaining the area. Then $I$ added to the area, the acces of the length over the width: 3,3. Moreover I have added length and width: 27 . Required length, width and area. [4]

Note. The Mesopotamians used sexagesimal number system, i.e. if $0 \leq a, b, c<60$, than $a, b ; c$, means $a \cdot 60+b+c \cdot 60^{-1}$. This transcription due to O. Neugebauer.(see e.g. in [4])

We can read a literal solution on the tablet, a sequence of calculations with the given numbers, without offering an explanation, or referring to some general rule.

Calculate as follows: add 27 the sum of the length and width to 3,3 , that is 3,30. Add 2 (sic!) to 27 you get 29. Halve this 29, 14; $30 \times 14 ; 30$ is 3,30; 15 . Substract 3, 30 from 3,30;15 get $0 ; 15$ and the square root of it is $0 ; 30$ Add this to the first $14 ; 30$, the length is 15 , and substract from the second $14 ; 30$, the width is 14. Substract the 2, that you have added to 27 , from the 14 , and the final width is 12 .

For the sake of convinience we transfer it into symbolic calculation parallel to the original one. We have to solve the 


$$
\begin{aligned}
x y+(x-y) & =3,3 \\
x+y & =27
\end{aligned}
$$

set of equations, where $x, y$ denote the length and the width, moreover let $y^{\prime}=y+2$.

$$
\begin{array}{ll}
27+3,3=3,30 & x y^{\prime}=3,30 \\
2+27=29 & x+y^{\prime}=29 \\
29: 2=14 ; 30 & \frac{x+y^{\prime}}{2}=14 ; 30 \\
14 ; 30 \times 14 ; 30=3,30 ; 15 & \left(\frac{x+y^{\prime}}{2}\right)^{2}=3,30 ; 15 \\
3,30 ; 15-3,30=0 ; 15 & \left(\frac{x+y^{\prime}}{2}\right)^{2}-x y^{\prime}=0 ; 15 \\
\sqrt{0 ; 15}=0 ; 30 & \sqrt{\left(\frac{x+y^{\prime}}{2}\right)^{2}-x y^{\prime}}=\frac{x-y^{\prime}}{2}=0 ; 30 \\
14 ; 30+0 ; 30=15 & \frac{x+y^{\prime}}{2}+\frac{x-y^{\prime}}{2}=x=15 \\
14 ; 30-0 ; 30=14 & \frac{x+y^{\prime}}{2}-\frac{x-y^{\prime}}{2}=y^{\prime}=14 \\
14-2=12 & y^{\prime}-2=y=12
\end{array}
$$

The reader can easily observe the permanent use of the methods al-jabr and al-muquabla. On the other hand it can be recognized that this method is almost the same as we use today.

The following two problems make the above evidence stronger. Namely, in the time of the Old Babylonian Empire (XVIIIth century BC) the Mesopotamian scibes used the methods al-jabr and al-muqabala frequently.

Problem 2. (Problem 2 in BM 13901) I have substracted the square fom the area and it is 14,30. (Here square and area mean the side and the area of a square.) [5]

The literal solution from the tablet:

1. Take 1 , the coefficient (of the square).

2. Divide it into two parts, $0 ; 30 \times 0 ; 30=0 ; 15$ you add to 14,30 .

3. The square root of 14,$30 ; 15$ is $29 ; 30$.

4. You add to $29 ; 30$ the $0 ; 30$ which you have multiplied by itself, 


$$
\text { "tmcs-klukovits" — 2011/5/16 — 12:52 — page } 150 \text { — \#4 }
$$

If we consider Problem 2 in modern notions, it calls us to solve a quadratic equation of the form of

$$
x^{2}-a x=b .
$$

Transforming the above literal solution into symbolic form we have

$$
\begin{aligned}
x^{2}-a x & =b \\
x^{2}-a x+\left(\frac{a}{2}\right)^{2} & =b+\left(\frac{a}{2}\right)^{2} \\
\left(x-\frac{a}{2}\right)^{2} & =b+\left(\frac{a}{2}\right)^{2} \\
x-\frac{a}{2} & =\sqrt{b+\left(\frac{a}{2}\right)^{2}} \\
x & =\frac{a}{2}+\sqrt{b+\left(\frac{a}{2}\right)^{2}}
\end{aligned}
$$

Here both methods are used again.

The next problem will show the use of al-jabr in its second meaning.

Problem 3. (Problem 7 in BM 13901) I add to the square multiplied by seven the area multiplied by eleven and it is $6 ; 15$. [5]

In modern termonology, the problem calls to solve the quadratic

$$
11 x^{2}+7 x=6 ; 15 \text {. }
$$

According to the literal solution written on the tablet, the first step was to multiply (the equation) by 11, than the further steps were as usual in that time. Now the solution step by step — but considering the reader's comfort — only in general with modern symbolism is as follow.

$$
\begin{aligned}
a x^{2}+b x & =c \\
a^{2} x^{2}+a b x & =a c \\
a^{2} x^{2}+a b x+\left(\frac{b}{2}\right)^{2} & =a c+\left(\frac{b}{2}\right)^{2} \\
\left(a x+\frac{b}{2}\right)^{2} & =a c+\left(\frac{b}{2}\right)^{2} \\
a x+\frac{b}{2} & =\sqrt{a c+\left(\frac{b}{2}\right)^{2}}
\end{aligned}
$$




$$
\text { "tmcs-klukovits" — 2011/5/16 — 12:52 — page } 151 \text { — \#5 }
$$

$$
\begin{aligned}
a x & =\sqrt{a c+\left(\frac{b}{2}\right)^{2}}-\frac{b}{2} \\
x & =\frac{1}{a}\left(\sqrt{a c+\left(\frac{b}{2}\right)^{2}}-\frac{b}{2}\right)
\end{aligned}
$$

\section{Conclusion}

As the above examples show, the two famous methods - al-jabr and almuqabala - that credited totally to Al-Khwarizmi, was known and used two thousand years earlier. But there is a remarkable difference. Namely, these methods as a technical skill was known only and used by the the Mesopotanian scribes of the Old Babylonian Empire. However they have never formulated them as a general rule that applicable for any equation. Al-Khwarizmi was the first scolar who write down them as a general rule. This fact does not decrease the merit of al-Khwarismi, but we must also provide some credit to the scribes of the Old Babylonian Empire.

\section{Algebraic methods}

The first European mathematician, who reached remarkable algebraic results, was Leonardo of Pisa. The second debated view was that between his Liber abaci (1202) and the bulk volume of Luca Pacioli entitled "Summa de arithmetica, geometria, proportioni e proportionalá" (1487) happened nothing remarkable in algebra, especially in solving cubic and biquadratic equations by algebraic methods.

The European scolars accepted the statement of the Moshlem mathematicians that these equations can be solved by geometrical methods only. This acceptance based on the neglection of several manuscripts and books. The first was Leonardo's Liber quadratorum (1225), the most substantial work of him, which was discovered as a part of a manuscript, the Trattato di practicha d'arismetica, composed by the Florentin master Benedetto in 1463. [3]

In the XIXth and XXth century several other manuscripts were found. Among them is a very important treatise, the Aliabraa argibra, composed by master Dardi of Pisa in 1344. In spite of the fact that some of them are contained in codices discovered in the middle if the XIXth century (e.g. Boncompagni and others), the careful examinations have started only in the second part of the last century. 
We could read some views on these algebraic treatises, but they were mostly sematic did not touched the real contents. Some typical sentence written by A. P. Juschkewitsch in the middle of the last century. [2]

- There are Italian manuscripts fom the centuries XIV and XV with some algebraic problems, but they dealt quadratic equations mostly.

- The unknown was called as cosa, its second power as quadrato censo or simply censo, and the higher powers as cubo, senso di senso,... (Probably in the works of Benedetto at the first time.)

- There were sporadic attempts to handle cubic equations algebraically without remarkable succes.

- There is a manuscript written by a really clever algebraist, but even his brilliance could not prevent to commit a lot of errors (probably he has refered here to the treatise of Dardi).

In this note we consider some questions from Dardi's manuscript, that sharply disprove the above and the similar opinions.

The treatise is known in three (mediaeval) Italian copies and one Hebrew translation. From the Hebrew version we know that the original was composed about 1344 in Pisa. The copies of the manuscripts are about 120 closely written leaves in an ample quarto size, i.e. about 270 (normal) pages each. And what is the most interesting, it contains a list of 198 different types of equations in rhetoric fashion. [1]

As a brief summary about it read a sentence from van der Waerden [6]: "This text is notheworthy for its unusual length, its adept handling of complex equations involving radicals and powers up to the 12th degree, and its correct solution of four irreducible cubic and quartic equations."

Consider first four typical equations from Dardi's list. The numberings due to Warren van Egmond's list. [1] For us the mediaeval rhetoric style is hardly understandable, therefore we use modern symbolic notation as Egmond did.

$$
\begin{aligned}
a x^{3}+c x & =b x^{2} \\
x & =\frac{b}{2 a} \pm \sqrt{\left(\frac{b}{2 a}\right)^{2}-\frac{c}{a}} \\
a x^{4} & =b x^{2}+n \\
x & =\sqrt{\sqrt{\left(\frac{b}{2 a}\right)^{2}+\frac{n}{a}}+\frac{b}{2 a}}
\end{aligned}
$$




$$
\begin{aligned}
a x^{3} & =\sqrt[3]{b x} \\
x & =\sqrt[8]{\frac{b}{a^{3}}} \\
\sqrt{a x^{3}} & =\sqrt[3]{b x} \\
x & =\sqrt[4]{\frac{b^{2}}{a^{3}}}
\end{aligned}
$$

Easy to see that the solution of equation (179) is false, the correct one is

$$
x=\sqrt[7]{\frac{b^{2}}{a^{3}}} .
$$

We have to point out that there are 3 incorrect solutions in the list only. Jushkewitsch did not examined the manuscript carefully enough.

The most interesting equations are the four inserted between nos. 182 and 183. These are one complete cubic and three complete quartic.

$$
\begin{aligned}
a x^{3}+b x^{2}+c x & =n \\
x & =\sqrt[3]{\left(\frac{c / a}{b / a}\right)^{3}+\frac{n}{a}}-\frac{c / a}{b / a} \\
a x^{4}+b x^{3}+c x^{2}+d x & =n \\
x & =\sqrt[4]{\left(\frac{d / a}{b / a}\right)^{2}+\frac{n}{a}}-\sqrt{\frac{c / a}{b / a}} \\
a x^{4}+c x^{2}+d x & =b x^{3}+n \\
x & =\sqrt[4]{\left(\frac{c}{4 a}\right)^{2}+\frac{n}{a}}-\sqrt{\frac{d / a}{2 b / a}} \\
a x^{4}+d x & =b x^{3}+c x^{2}+n \\
x & =\sqrt[4]{\left(\frac{c}{4 a}\right)^{2}+\frac{n}{a}}-\sqrt{\frac{d / a}{2 b / a}}
\end{aligned}
$$

For the first glance, the solutions are false in this general form. But if we consider the original problems we have to change our mind. All equations are presented in rhetoric style as special concrete problems. Therefore if we rewrite the problem as an equation, all the coefficients are definite numbers not mere symbols as above or in Egmond's paper. 


$$
\text { "tmcs-klukovits" — 2011/5/16 — 12:52 — page } 154 \text { — \#8 }
$$

Consider first the cubic - equation (E1) — in its original form. It was

$$
x^{3}+60 x^{2}+1200 x=4000,
$$

that would originated in certain practical problem. In Meadiaeval manuscripts (e.g. certain "handouts" for masters) we often find loan problems. One typical can be read in a Trattato d'abaco written by the famous reneisance painter Piero della Francesca.

Lonn Problem. Someone lends to another one 100 Lira, and after 3 years receives 150 Lira with annual capitalization of the interest. One asks at what monthly rate of interest the loan was given. [6]

According to the original solution, we have to express the monthly rate of interest in denarii pro Lira (one Lira is $20 \times 12$ denarii). If we denote it by $x$, then we get the equation (in modern symbols)

$$
100\left(1+\frac{x}{20}\right)^{3}=150
$$

which is the same as Dardi's equation (E1').

Before going to further analysis we mention, that the Dardi's concrete version of (E2) can be obtained from a quite similar loan problem if the creditor gets 160 Lira after 4 years.

The question is, how to find Dardi the solution given in (E1). Unfortunately definit answer probably never will be known, therefore we have to look for some possible reconstructions.

One possibility is as follows. We can suppose, that Dardi knew the famous algebra book of Al-Khwarismi. The Moshlem mathematician used a square to interpret or proove his method of solving quadratic equations. We can imagine that Dardi used a special cube for getting a solution of some cubic.

After van der Waerden [6] consider the following cube and decompose it into 8 parts.

- a cube with side $x$,

- 3 prisms with square base $x$ and height $L$,

- 3 prisms with square base $L$ and height $x$,

- a cube with side $L$. 


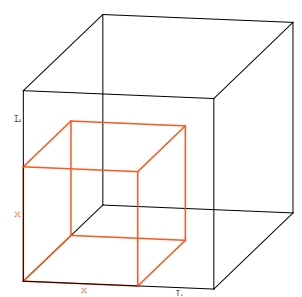

The volume of the big cube is

$$
(x+L)^{3}=x^{3}+3 x^{2} L+3 x L^{2}+L^{3} .
$$

Comparing (C) with equation (E1) in case of $a=1$ we have

$$
\begin{aligned}
x^{3}+3 x^{2} L+3 x L^{2}+L^{3} & =(x+L)^{3} \\
x^{3}+b x^{2}+c x & =n .
\end{aligned}
$$

This two equations will be the same if

- we add $L^{3}$ to both sides,

- and the following is satisfyed

$$
\begin{aligned}
& c=3 L^{2} \\
& b=3 L
\end{aligned}
$$

or in the other hand

$$
\begin{gathered}
L=\frac{c}{b} . \\
(x+L)^{3}=n+L, \\
x=\sqrt[4]{n+L^{3}}=\sqrt[3]{\left(\frac{c}{b}\right)^{3}+n}-\frac{c}{b} .
\end{gathered}
$$

REMARK 1. The above method is not general, it works with special coefficienst only.

REMARK 2. For the first glance, it would be more simple to apply the binomial theorem, but this is less probable, because this theorem was not widely known that time in Europe. On the bases of the Moshlem traditions the geometrical methods were dominant in the cases of cubic. 


$$
\text { "tmcs-klukovits" — 2011/5/16 — 12:52 — page } 156 \text { — \#10 }
$$

The last two equations (E3') and (E4') should be originated in a problem of Dardi.

Problem D. To divide 10 into two parts such that their product divided by their difference is a given number (more exactly, the square root of a given number).

Dardi solved the problem with $\sqrt{18}$ and $\sqrt{28}$. In the first case we have the equation

$$
\frac{x(10-x)}{x-(10-x)}=\sqrt{18} \text {. }
$$

Taking the square of both sides we get

$$
x^{4}-20 x^{3}+28 x^{2}+720 x=1800,
$$

which is Dardi's (E3'). (In case of 28 we could get equation (E4')) As the solution of (E3') (or (7)) in case of $a=1$ Dardi's suggestion is the formula

$$
x=\sqrt[4]{\left(\frac{c}{4}\right)^{2}+n}+\frac{b}{4}+\sqrt{\frac{d}{2 b}},
$$

or in our special case

$$
x=\sqrt[4]{\left(\frac{28}{4}\right)^{2}+2800}+\frac{20}{4}+\sqrt{\frac{720}{2 \cdot 20}} .
$$

Van der Waerden in [6] gave a very nice procedure how could find Dardi this result. As a starting point consider (1) as a quadratic equation ( $g$ denotes the given number).

$$
\begin{aligned}
\frac{x(10-x)}{x-(10-x)} & =\sqrt{g} \\
10 x^{2} & =(2 x-10)^{2} \sqrt{g} \\
x^{2}-2(5-\sqrt{g}) x & =10 \sqrt{g}
\end{aligned}
$$

The well-known solution of it is

$$
\begin{aligned}
x & =5-\sqrt{g}+\sqrt{(5-\sqrt{g})^{2}+10 \sqrt{g}} \\
& =5-\sqrt{g}+\sqrt{25+g}
\end{aligned}
$$




$$
\text { "tmcs-klukovits" — 2011/5/16 — 12:52 — page } 157 \text { — \#11 }
$$

On the other hand squaring (1) (with $18=g$ )

$$
\begin{aligned}
x^{2}(10-x)^{2} & =g(2 x-10)^{2} \\
x^{4}+(100-4 g) x^{2}+40 g x & =20 x^{3}+100 g
\end{aligned}
$$

Compare it with (E3) $(\mathrm{a}=1)$

$$
x^{4}+c x^{2}+d x=b x^{3}+n
$$

and we have

$$
b=20, \quad c=100-4 g, \quad d=40 g, \quad n=100 g .
$$

The crucial point is how we (or Dardi?) can write the (6) solution in the form of (3), which is similar to the solution of (E1) that can be obtained in geometric way. In the case of biquadratic equations there are no traditional geometric way. Van der Waerden supposed that Dardi wants to have some similar form (of the solution) in this case, too.

Consider the terms in (6) taking into account the Problem D (shortly D).

- The first term is 5 , which is the half of the 10 in $\mathrm{D}$, while the $b=20$ is $2 \cdot 10$. Therefore the first term of (6) is $\frac{b}{4}$, the second term of (3).

- The second term in (6) is $\sqrt{g}$, where $q=18,28$, while in (8) $d=40 \mathrm{~g}$. If we devide $d$ by $2 b=40$, then get $g$, thus the third term of (3) is

$$
-\sqrt{\frac{d}{2 b}}=-\sqrt{g} .
$$

- To get the first term of (3) remember the (supposed) Dardi's aim. We (he) want (wanted) a formula similar to the cubic, where the form of the first term is

$$
\sqrt[3]{\text { something }+n}
$$

( $n$ is the constant term of the the equation), therefore the 4 th root of "something plus $n$ " must be here. We (he) reach (reached) it, if the "something" is equals to $\left(\frac{c}{4}\right)^{2}$. If it is so, then

$$
\begin{aligned}
\sqrt[4]{\left(\frac{c}{4}\right)^{2}+n} & =\sqrt[4]{(25-g)^{2}+100 g} \\
& =\sqrt[4]{(25+g)^{2}}=\sqrt{25+g}
\end{aligned}
$$

which is the third term of $(6)$. 


$$
\text { "tmcs-klukovits" — 2011/5/16 — 12:52 — page } 158 \text { — \#12 }
$$

Conclusions

1. We have to emphasize that all above could be formulated on the basis of a hypothesis due to van der Waerden. We do not know the original concepts of master Dardi. Our way of thinking might be similar to that of Dardi, but no more.

2. We have to stress that master Dardi's manuscript can be considered an important step towards to the algebraic solution of cubic and biquadratic equations. Therefore all opinions do not share this view are hardly questionable.

\section{Acknowledment}

The author is grateful to Professor Edmund Robertson for his comments and suggestions.

\section{References}

[1] W. van Egmond, The algebra of Master Dardi of Pisa, Historia Mathematica 10 (1983), 399-421.

[2] A. P. Juschkewitsch, Geschichte der Mathematik im Mittelalter, Teubner, Leipzig, 1964.

[3] L. T. Rigatelli, Documenti per una storia dell'algebra in Italia dal XIII al XVI secolo, La Storia delle Matematiche in Italia, Cagliari, 1982, 333-339.

[4] B. L. van der Waerden, Science Awakening, Oxford University Press, New York, 1961.

[5] B. L. van der Waerden, Geometry and Algebra in Ancient Civilizations, Springer-Verlag, Berlin Heidelberg New York Tokyo, 1983.

[6] B. L. van der Waerden, A History of Algebra, Springer-Verlag, Berlin Heidelberg New York Tokyo, 1985.

LAJOS KLUKOVITS

UNIVERSITY OF SZEGED, BOLYAI INSTITUTE

ARADI VERTANÚK TERE 1.

H-6720 SZEGED, HUNGARY

E-mail: klukovits@math.u-szeged.hu

(Received March, 2011) 